\section{Das Senf-Beifuß-Syndrom}

Eine IgE-vermittelte Senfallergie ist bisher nur selten beschrieben worden. Doch vor allem aufgrund von Kreuzreaktivitäten kommt dieser Nahrungsmittelallergie eine größere Bedeutung zu als bisher angenommen.

E. in in Forscherteam aus Las Palmas auf Gran Canaria rekrutierte für eine prospektive Studie Patienten mit selbst berichteter Senfallergie und Symptomen wie orales Allergiesyndrom, Urtikaria/ Angioödem und Anaphylaxie. Bei den insgesamt gefundenen 38 Probanden sollten verschiedene doppelblinde plazebokontrollierte Nahrungsmittelprovokationen (DBPCFC) sowie Pricktests mit Aeroallergenen und Nahrungsmitteln erfolgen. Die Ergebnisse wurden mit denen bei gesunden Probanden verglichen.

Von den 38 Patienten unterzogen sich 24 dem DBPCFC-Test mit Senf, eingearbeitet in eine gelb gefärbte und mit Vanille und Zitrone aromatisierte Joghurt-Grundlage. Bei 14 Patienten verlief der Test positiv, ein positives Testergebnis korrelierte mit einer grö-
Beren Reaktionsfläche im Pricktest. Als Cut-off-Wert für einen positiven Hauttest mit einem kommerziell erhältlichen Senf-Extrakt wurde ein Quaddeldurchmesser von $8 \mathrm{~mm}$ errechnet, die Spezifität betrug $90 \%$ und die Sensitivität $50 \%$.

Bei 97,4\% der Patienten mit einer Senfallergie lag gleichzeitig eine Sensibilisierung gegen Beifußpollen vor mit partieller Kreuzreaktivität bei „RAST“Inhibitionstests. Außerdem wiesen alle Patienten Sensibilisierungen gegen andere Nahrungsmittel aus der Familie der Brassicaceae auf - neben Senf gehören z. B. die verschiedenen Kohlsorten einschließlich Brokkoli sowie Rettich und Radieschen in diese Familie. Auch bestanden bei vielen der Probanden Sensibilisierungen gegen Nüsse (ohne
Erdnüsse, 97,4\%), Leguminosen (mit Erdnüssen, 94,7\%), Getreide (78,9\%) und Rosaceae-Früchte (89,5\%). Etwa $40 \%$ der Patienten mit Nahrungsmittelsensibilisierungen hatten Symptome, sechs der Patienten hatten bereits eine anstrengungsinduzierte anaphylaktische Reaktion erlitten.

Kreuzreaktivitäten zwischen Nahrungsmitteln untereinander sowie mit Aeroallergenen sind ein bekanntes Phänomen. Klinisch bedeutsam in Mitteleuropa ist z. B. das "Sellerie-Beifuss-Gewürz-Syndrom “. Für die in der vorliegenden Studie gefundenen Zusammenhänge schlagen die Autoren den Namen „Beifuß-Senf-Allergiesyndrom“ vor.

Fazit: Eine Senfallergie kann zu schweren anaphylaktischen Reaktionen führen und ist häufig vergesellschaftet mit Sensibilisierungen gegen andere Nahrungsmittel sowie Beifußpollen.

$b k$

Figueroa J et al. Mustard allergy confirmed by double-blind placebo-controlled food challenges: clinical features and crossreactivity with mugwort pollen and plantderived foods. Allergy 2005; 60: 48-55

\title{
Zur Milben- noch die Garnelenallergie?
}

\section{Extrakte zur spezifischen Immuntherapie (SIT) enthalten neben} den Allergenen, gegen die hyposensibilisiert werden soll, noch zahlreiche weitere Proteine. Diese können potenziell neue unerwünschte Sensibilisierungen auslösen. Ein Milbenextrakt wurde daraufhin näher untersucht.

talienische Forscher gingen der Frage nach, ob es nach einer SIT bei Hausstaubmilbenallergie de novo zur Entwicklung einer Garnelenallergie kommen kann. Als kreuzreaktives Allergen kommt Tropomysin in Betracht, ein Protein der Wirbellosenmuskulatur, das gleichermaßen sowohl in Milben als auch in Krustazeen und Mollusken vorkommt.

An der Studie nahmen 70 Patienten mit einer Hausstaubmilbenallergie teil, die zu Beginn alle regelmäßig und problemlos Meeresfrüchte zu sich nahmen. Davon erhielten 31 über 3 Jahre eine SIT mit einem Depot-Hausstaubmilbenextrakt des Unternehmens Aller-

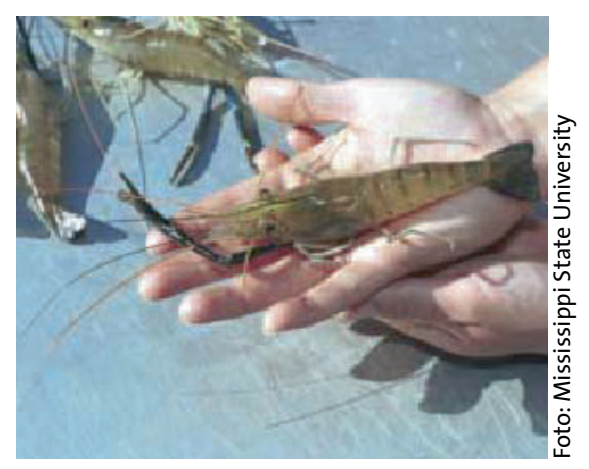

Das Hauptallergen der Garnele Pen a 1 ist das Muskelprotein Tropomyosin und dieses kommt auch in der Hausstaubmilbe vor - eine potenzielle Quelle für Kreuzreaktionen. gopharma, die übrigen 39 dienten als Kontrolle. Die Nachbeobachtungszeit betrug mindestens 3 Jahre. Eine mögliche Sensibilisierung wurde sowohl im Hautpricktest als auch im oralen Provokationstest mit frischen Garnelen überprüft. In keiner der Gruppen entwickelte ein Patient eine Nahrungsmittelallergie gegen Garnelen und auch andere Meerestiere wurden nach Angaben der Patienten weiter problemlos vertragen.

Fazit: Eine spezifische Immuntherapie mit einem Hausstaubmilbenextrakt scheint - zumindest bei dem in dieser Untersuchung verwendeten Depotpräparat - keine neue Sensibilisierung gegen Krustazeen-Tropomyosin nach sich zu ziehen.

Asero R. Lack of de novo sensitization to tropomyosin in a group of mite-allergic patients treated by house dust mitespecific immunotherapy. Int Arch Allergy Immunol 2005; 137: 62-5 